\title{
The Analysis of Basic Communication Process Based on the Knowledge Network ${ }^{1}$
}

\author{
Yin Wensheng ${ }^{\mathrm{a}}$, Qin Weiwei ${ }^{\mathrm{b}}$ \\ School of Mechanical Science and Engineering, \\ Huazhong University of Science and Technology, Wuhan 430074, China \\ awsyin@mail.hust.edu.cn, ${ }^{\mathrm{b}} \mathrm{m} 201470304 @$ hust.edu.cn
}

Keywords: knowledge communication; knowledge network; semantic network; standard model; knowledge component

Abstract. The diffusion and application of knowledge can not be separated from knowledge communication. In this paper, the communication process of knowledge is abstracted into the communication process between the agents that realized through a specific or abstract medium which is called as the knowledge network. Knowledge network is the graphical representation of knowledge and can be abstracted as the basic graphic elements and basic functional components. The former is the node and the connection, the latter is the knowledge components constructed with the node and the connection to express some semantic. In order to realize the knowledge communication, firstly, the semantic consistency of each node and the connection in the knowledge network should be satisfied, that is to say we need to establish authoritative knowledge dictionary, secondly, design better structure of knowledge components to describe and apply knowledge better. Finally, in order to improve the accuracy and convenience of the knowledge communication, we must design a suitable comparison and creation method of knowledge network which based on black box theory. A comparison of two kinds of knowledge networks is made on the semantic network and standard model of knowledge representation. The former is simple but not good for representing complex semantics. The latter can not only represent complex semantics in a single knowledge element, but also can be easily combined by the knowledge network. At the same time, it can be easily to carry out the hierarchicalization and partition of knowledge network, and the formed knowledge network can be computed, so it can meet the communication process of knowledge network better.

\section{Introduction}

Knowledge acquisition, knowledge innovation and knowledge application are intelligent activity of agents. Whether the thinking activity of a single agent, or the cooperative work of a number of agents, can not be separated from a specific or abstract knowledge representation. Because knowledge is the representation of things and their relationships, it can be represented by graphs, so the knowledge representation forms a complex network, called knowledge network. Obviously, it is very important to study the mechanism of knowledge network for the studying of artificial intelligence.

\section{Related Work}

Human knowledge activities can be divided into two major categories: independent thinking and mutual communication. Independent thinking refers to a single person's thinking activity, that is, in a person's head or with other non intelligent body to complete the knowledge activity, while the mutual communication refers to the exchange of ideas among many people. There are various forms of communication, such as conversation, class, speech, telephone, etc. It is assumed that the agent represents knowledge with the knowledge network, then the independent thinking can be reduced to the mutual communication, only the object to be exchanged is virtual one. Therefore, human

\footnotetext{
${ }^{1}$ National Natural Science Foundation of China: Assembly sequence planning based on the knowledge of engineering connection (51175200)
} 
knowledge action can be abstracted into a kind of communication, that is, the interaction between the knowledge networks of agents.

The common forms of knowledge representation language includes: state space representation, predicate logic, ontology, semantic representation, frame representation, production, Petri net, database, computer program, object oriented representation, etc.[1-11] No matter what means, can be abstracted into the relationship between certain nodes and connections, can be expressed as some of the network structure, which is known as the cause of the network of knowledge.

The knowledge representation and processing method are analyzed from the perspective of the interaction between agents as follows.

Formalization of Knowledge. Natural language is widely used in knowledge representation and communication, but it is very difficult to learn, understand and apply it for non-human. So a natural way is to design a language which can be understood by computer. It is believed that in natural language there are a large number of multi valued, abbreviation, implicit, metaphor and so on, and formal language is very strict, there is no ambiguity. Firstly, people proposed that using predicate logic and other methods to express knowledge and carry out the proof of the theorem.

Predicate logic is abstract, which can guarantee the accuracy of true value operations, but it can be a problem for the semantics of natural language. In the daily communication of human, we needs a lot of common sense, each thing is not a causal relationship, but the related relationship, these are difficult to deal with for predicate logic.

Semantic Association. In order to express semantic relations, we must introduce the knowledge of psychology and linguistics, and discuss the basic principles and methods of human communication.

The association theory is considered to define the object by means of an association between an object and other objects. Any object or concept is the part of the whole world knowledge. When making the perception or thinking of an object, the concept is first established, then the concept is connected by a certain relationship with other concepts, namely knowledge can be represented by connecting all the concepts with different connections. This method is called semantic network.

First of all, concepts and relationships must be determined when using the theory of association to represent the knowledge. As the related relationship is also a concept, so in the discussion followed it is replaced with concept. Obviously, the concept itself is difficult to determine. Two people, even though they use the same language, there is also a difference in the concept of one thing.

In order to ensure that different people have the same understanding of the same thing, it is necessary to specify some primitive to express the concepts in the knowledge system. For example, in a natural language, there are many words are defined. In these words, it can mainly be divided into two categories: nouns and predicates. The noun is used to express the concept, and the predicate expresses how things change, that is, the related relationship is described. Obviously, because of the objective things and their correlation, the number of nouns and predicates is huge. So we must reduce the number of predicates, and classify nouns.

Secondly, the analysis of structure of semantic network is needed. How to describe the concept and the relationship among the concepts needs a careful study. On the one hand, the relationship among them is various, and on the other hand, the number of them is enormous. The semantic network should be as simple as possible, at the same time it should have strong semantic expression ability. The general practice is to divide the knowledge into different levels and different partitions, which is convenient for management, and also greatly improve the efficiency of knowledge solving.

Fast Association. People need a lot of background knowledge and common sense in the process of communication. If you search for all the knowledge you need to spend a lot of time. Through the analysis of human activities, it is found that human beings actually only search for the knowledge network of some parts, and even only use the knowledge directly, and therefore, a lot of methods of associate knowledge are put forward.

Script is a fast method to associate knowledge. It represents the knowledge in a particular context by recording a fixed sequence of events in a particular context (Schank and Abelson, 1977)[12] .This method is very efficient, and it is not easy to make mistakes, it is a common method for human beings. 
The defect of the script method is that when the environment changes, it must be changed in time. Obviously, the effect is very good if you consider the problem carefully in the design script.

Frame representation is another fast method of associating knowledge. It can be understood as the improvement of the script method. It not only can obtain the knowledge of the fixed structure, but also can change the part of the change. When things do not coincide with the definition of the framework, you can refer to a certain frame, and then modify some of them. You can express complex things by layering and combining the framework.

Object Oriented Method. The fast association method is the method that the human often uses, or it may be the more general method of knowledge representation. From the frame representation, knowledge is a multi frame structure, each frame can be expressed as a kind of thing, it has a groove, can express the attribute of things. The framework is inherited and can be inherited as an example of a class framework. Therefore, the object oriented method is put forward for the further development. Other Knowledge Representation Method. In addition to represent by the symbols, knowledge can also be expressed and applied in other ways, such as a kind of physical structure. In the field of artificial intelligence, the other two kinds of famous knowledge representation methods are connectionism and behaviorism $[13,14]$.

\section{Knowledge Communication Process}

Human knowledge can not be copied, must be acquired through learning. Similarly, various ideas in the human brain must be mapped out for the application through various activities. A variety of human activities, in essence, is the communication of knowledge. Activities among people and people are knowledge communication activities, and the activities among people and all kinds of things can also be seen as knowledge communication activities, that is, knowledge application activities. Knowledge communication activities can be divided into two categories: knowledge acquisition and knowledge application activities.

Hypothesis. Hypothesis 1: there are some physical or logical knowledge networks in each agent, and the knowledge communication between them is conducted by a physical or logical knowledge network.

According to ontology, knowledge represents things and their relationship, according to the theory of graph theory, it is a kind of network. Regardless which method is used, the network can be called knowledge network.

The agent here mainly refers to two categories: human and artificial objects, do not consider animals and other things. Obviously, there are cognition about the things and their relations in the world in the agent, in the form of a physical or logical. Therefore, it can be said that the knowledge communication between two agents is a kind of communication between two pieces of knowledge network, which are similar at some degree, or the knowledge communication between the agents is realized by some kind of knowledge network.

Because the physical knowledge network can be represented by a logical knowledge network, the knowledge communication between the agents is carried out through a logical knowledge network. In a simple way, the knowledge communication between the agents is through knowledge network.

Whether the method used in the knowledge network of the two agents is the same, it can be used in the same logic, but it may be different corresponding to the meaning of things and their relations in knowledge networks.

The Premise of Success. Hypothesis 2: if the communication of two agents is successful, then there must be corresponding part in their knowledge network.

According to hypothesis 1, two agents communicate with each other by the knowledge network, and only when there is some corresponding part, they may understand each other's semantics.

In addition to artificial intelligence, generally speaking, the two agents are not likely to be completely consistent, it is only possible to achieve some of the same, that is, the knowledge network of the two agents can not be completely consistent even in logic, for example, the knowledge network in a smart agent may be more complex than other one, but if there is a same part, they can achieve knowledge communication. 
In other words, in order to make the knowledge communication successful, there must be a consensus of the knowledge network of the two agents.

Here, we need to explain that the knowledge networks of two agents are consistent but not equal. For human communication, this is very obvious, even if you get the same information, what you think is likely to be different.

In order to facilitate the discussion, we extended the communication activities, that is, a person's thinking activities or the use of an artificial intelligence body can also be understood as knowledge communication activities, only the communication object is a virtual agent. In order to facilitate the following instructions, all having no intelligent things such as book can be abstracted into agents.

Operation of Knowledge Network. Suppose there are two agents, and each has its own knowledge network. Their knowledge networks are represented by the same method, although they may be gotten through a certain way of the conversion process. For example, a Chinese and an American, they speak their own language, may use different expressing method of knowledge networks, but at this time we assume that all have been converted into the same and abstract semantic network representation of knowledge network. It is worth noted that the representation method of knowledge network is the same dose not means that the element of knowledge network is same. For example, two Chinese people are using Chinese to communicate, but the same words may not be exactly the same in each person. It also shows that the success of the communication is actually the consistence not the equal of the knowledge network.

Human communication mainly exists in the following activities: narration, exclamation, questions, identity, etc. Apart from the emotional aspects of things, it can be divided into two categories: one is to tell others the knowledge, and another is to get knowledge from others. Corresponding to the change of knowledge network, it can be summed up in two categories of creating knowledge network and computing knowledge network.

Creating Knowledge Network. It is to create, modify and delete the knowledge network in the agent according to the knowledge acquired by the communication with other agents. Here, simply call the creation, modification and deletion of knowledge network as create activities.

Computing Knowledge Network. It is to compute the knowledge network in the agent and get new information according to the information obtained by the exchange of information with other agents.

The Function of Intelligence. The process of knowledge communication of two agents is actually the process of the different knowledge networks come into same. So, for the non-artificial agent, the consensus of the knowledge network not only includes the similarity of the graph, but also the content of the nodes and the connections in the knowledge network.

Therefore, intelligence must exist in the knowledge communication of non-artificial agent. The main role is as follows.

1) Establish the various elements of the knowledge network graph, such as nodes and connections, as well as the corresponding relationship between them.

2) Establish knowledge network. How to build the knowledge network according to the information acquired by the knowledge communication and make the two knowledge networks have a similar relationship.

3) Knowledge network solution or reasoning. It is more advanced processing and application of complex knowledge network. Knowledge solving or reasoning generally refers to get new knowledge based on the existing knowledge. Such knowledge resolving or reasoning is different with computing knowledge network. It includes the process of creating the knowledge network, and not just the computation of the knowledge network. In fact, the process of creating a knowledge network is a highly intellectual activity, and the computing knowledge network is just a kind of fixed computing process. So the intelligence is mainly embodied in the process of creating the knowledge network in knowledge communication activity. 
When there is only one agent, it can also carry out knowledge communication activities, but its information comes from the same agent, the knowledge communication can be understood as an agent and its copy.

In short, intelligent function of agent is to construct the knowledge network within the agent, and to keep the knowledge network consistent with each others

\section{Structure and Function of Knowledge Network}

From the above analysis, it can be known that the key to realize the knowledge communication is the consistent operation of knowledge network, and the most critical is how to construct the knowledge network. According to this view, the methods and characteristics of the knowledge network constructed by semantic network representation and knowledge representation standard model will be discussed as follows.

Basic Requirements. Taking the natural language as the reference, according to the assumption of the successful communication of knowledge, the following conditions must be satisfied.

1) Using the same knowledge network representation method

The comparison of same knowledge network representation method is easy to implement, otherwise it will need to be converted. For example, English and Chinese must be translated to understand their meaning.

2) Define a consistent knowledge dictionary

For the knowledge represented by the same language, if the vocabulary and usage is inconsistent, it also brings communication barriers. For example, there are some differences between American English and British English, and a Chinese in the South and another in the North China have different vocabulary, etc.

3) Build a clear knowledge network

In the natural language, the article with clear structure is easier to understand than the article with logical confusion. And similarly, the knowledge network with clear structure is also easy to express knowledge.

4) Construct the similar knowledge network as much as possible

Obviously, the expression way to the same knowledge is various. A proposition composition, even if all students of country to write, it may not appear exactly the same composition. The process of writing composition is the process to describe the knowledge network. Each person's knowledge network is different, so the method described is different, the final results are different. This is also the reason why natural language is difficult to understand. In order to overcome this problem, in fact, we often provide format documents, such as computer application system development documents, which are required to follow some certain rule for understanding and communicating easily.

5) Make knowledge networks computable

The purpose of the creation of knowledge network is to carry out the computation of the knowledge network. Only through the computation of the knowledge network can we obtain the knowledge. If the knowledge network is not computable, the function of the additional knowledge is needed, which will greatly affect the efficiency of the application.

By further analysis of the process of knowledge communication, we can know:

1) Most of the knowledge networks of the human brain are dynamic

The human brain need to deal with a lot of things every day, the amount of knowledge involved is very large, many of them have not experienced before. If it is static knowledge network, it is very large and becomes impossible. At the same time, it is also bad for reasoning, because it will take more time. However, there still are some relatively static knowledge networks in the human brain, such as strengthening training is to create a static knowledge network and to speed up the solving of knowledge.

2) Knowledge network is divided into partitions and layers

The knowledge network of human brain is very large. In order to improve the efficiency, it is needed to deal the knowledge network with the partitioning and hierarchical processing. On the one 
hand, it is easy to manage related knowledge. On the other hand, it can greatly improve the reasoning speed.

3) There are class model and individual model

Knowledge networks in the human brain can handle both class and instance knowledge. Obviously, class knowledge and instance knowledge are needed in knowledge solving. The operation object of knowledge solving is usually an instance object, and the result of the operation is also an instance object, but in the knowledge management, knowledge is usually in the form of a class model, which can greatly reduce the complexity of the knowledge network.

Knowledge Dictionary. In the knowledge representation of the standard model of knowledge network, the most basic element is the input, output and processing of the model, and the input and output parts are correspond to the thing or concept, and the processing part is to describe the relationship between these things. These elements are called words in the language.

Obviously, for any non-artificial agent, there are differences in the understanding of any two agents to the objective things, that is, the semantic differences of the corresponding words in the knowledge network of the two agents are different. For example, the sun in the eyes of John and Smith is likely to appear differences, John may only understand some natural characteristics of the sun and Smith may understand the spectrum characteristics of the sun. The existence of these differences is closely related to the background, life experience, education, and the degree of knowledge of the subject.

In general, no matter what kind of knowledge representation method is used, it can be represented by a kind of knowledge network, and their basic elements are the basic vocabulary. Therefore, in order to ensure the success of knowledge communication, a standard knowledge dictionary must be established, which provides the meaning of each word and the basis for the construction of the semantic of each knowledge network.

Knowledge dictionary is a kind of agreement, the meaning and usage of each word is determined in the dictionary. Latin letters are used in many countries in the world, but it is different for different languages. Of course, some words can also be related to the meaning of the word, such as the letter "O" can correspond to the mouth shape and many words in Chinese characters are conformity between shape and meaning.

Knowledge dictionary is very important for knowledge communication. As knowledge is exploding, new words are emerging, and the knowledge dictionary must be amended and abided by the users.

The Requirements of Basic Functional Structure. In the knowledge network, the node and the connection can not represent a complete knowledge. The basic functional unit of knowledge network, which is formed by the combination of nodes and the connections, is usually called the knowledge component, which is the smallest functional unit of the knowledge network. Different knowledge representation methods have different knowledge component definitions. In the semantic network, two nodes and the connections between them constitute a knowledge component. While in standard model knowledge network, a complete model constitutes a knowledge component. In order to meet the requirements of knowledge communication, knowledge component should have the following characteristics:

Simple structure

With sufficient descriptive power

Facilitate to knowledge operation

Facilitate to the comparison of different knowledge networks

There are many traditional knowledge representation methods, but most of them can use the semantic network method to express, such as predicate logic, generative method, frame representation method, etc. While the state space method, Petri net and computer program are similar to the standard model. The structure of knowledge network is discussed by comparison of the semantic network and standard model as follows.

Structural Features of Knowledge Components. Obviously, for a single piece of knowledge or semantic, the knowledge component structure of semantic network method and standard model are 
very simple and semantic network method more simple, it is often only describe the relationship between two nodes and is the most simple method in all knowledge network. While the standard model describes the relationship between multiple things and their relationships, so the input and output of the content can be a lot.

Descriptive Ability. In the knowledge system, we generally assumed that the predicate is indivisible, and knowledge component must contain a predicate. The knowledge component of the semantic network is very simple. A knowledge element can only describe a simple knowledge, which is corresponding to a simple sentence in a natural language generally. In order to describe the complex semantics, a combination of multiple knowledge components must be carried out. Although the knowledge components of standard model only contain a predicate, it can describe more input, output, and the input and output is also allowed to change, so that it can describe the more complex semantics. At the same time, the standard model can be combined to solve more complex problems. We discuss descriptive ability from the semantic complexity, reusability, and ambiguity as follows.

1) Complexity

For the description of complex semantics, there are two methods, one is to increase the semantic description of a single knowledge component, and the other is to combine the knowledge components. In natural language, the complex semantics is also described in two ways, by means of complex sentences or multiple sentences.

Because the semantic network can only describe the relationship between two nodes, it can not improve the descriptive ability of knowledge components by increasing the descriptive ability of knowledge components, and can only take a combination for complex things. In other words, the relationship among large number of things in reality can only be expressed by a combination of simple knowledge components. For example, in the semantic network of Simon, in order to define the meaning of the statement "John sent Marie 999 red roses", it is necessary to construct a semantic network as shown in Fig. 1. And with the increase of the complexity of the semantic, the construction of the semantic network becomes more complex, which is difficult to operate, even for people. So the semantic network is suitable for the relatively simple semantic representation, and generally as the application of static knowledge network.

For the standard model, there are two methods can be used to express complex semantics. On the one hand, it can express the complex relation between multiple objects by setting up complex input and output information, and using the knowledge component to express complex meaning. On the other hand, it can be expressed by the series and parallel operation of knowledge components to express complex semantics.

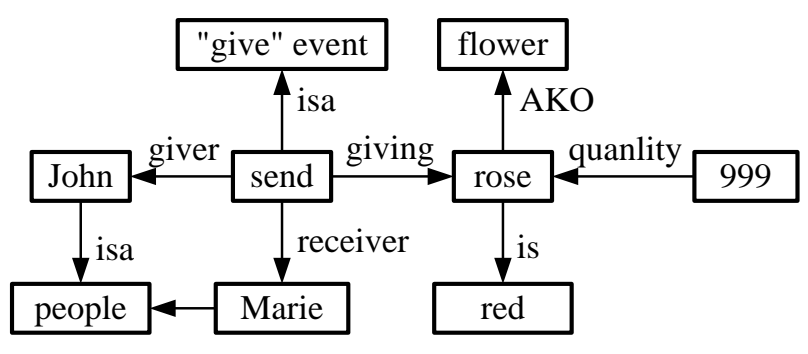

Fig. 1 Simmons's semantic network These two methods all have better operability, and the knowledge network is easy to understand, and it is easy to apply for knowledge.

2) Reusability

Knowledge components and knowledge networks are all reusable. The general assumption is that in the knowledge network, all nouns should be consistent, reuse mainly refer to the reuse of predicate. For example, some of the relationships in the semantic network can be applied in many areas of the knowledge network, and the standard model can also be used in the knowledge network. The reuse of knowledge components and knowledge networks can be divided into two aspects, and one is the reuse of basic components (i.e. predicates), another is the reuse of knowledge networks. In the semantic network, the predicate is expressed as a line, and the reuse of basic component is easy. But for the reuse of a piece of knowledge network, it is needed to abstract and re-combine the content of the block, for it is difficult to define a piece of semantic network as a component. For the standard model, the reuse of a basic component is simple, and a piece of knowledge network is also simple, all we 
need is to build a new model and define the model and the contact with the outside world as input and output.

3) Ambiguity

In the semantic network, it is as much as possible to define the relationship among the various things for inference, but may cause ambiguity. For one reason is that knowledge networks are complex and difficult to understand. In addition, it is impossible to define all of the relationships. Some of the relationships are identified in reasoning. Different reasoning machines will probably get different results. For example, the semantic meaning of Fig. 1 can be "John sent Marie 999 red roses", but, if we take the right part, it may be "roses are red", but rose is not only red, may have white roses, blue roses, etc.

In the standard model, if the processing part is determined, the situation of the input determines the results of output generally, so it will not produce ambiguity. Of course, on the other hand, the standard model of knowledge network can not express other knowledge, a knowledge network can only represent a specific knowledge, if you want to express other knowledge, and you must re-create a new knowledge network.

\section{Operation of Knowledge System}

Knowledge network is the representation of knowledge, which is not static, but dynamic change, that is, in the process of the operation of the knowledge system, the creation, modification, deletion and operation of knowledge network is carried out constantly. The operating process of the knowledge system is the activity of the agent. Here, we discuss the operating process and processing method of the knowledge system, which are mainly concerned with the processing of computation and comparison of knowledge networks.

Knowledge Network Computation. The purpose of creating the knowledge network is able to carry out the computation of knowledge network. Only through the computation of knowledge network can we get useful information. In the study of artificial intelligence, on the one hand, the research is on how to express knowledge, on the other hand, on how to realize the reasoning.

In the traditional knowledge representation, the representation of knowledge is not directly related to the computation of knowledge networks. For example, predicate logic is merely a representation of knowledge, other logical computations and methods are required for the knowledge solution. Similarly, the semantic network method mainly describes the relations between concepts, and needs some relevant inference rules. Obviously, the traditional knowledge solution not only contains two parts of knowledge network construction and knowledge network computation, but also contains some intelligence, although the solution is designed by man, but we think that the machine has smart generally.

In the knowledge network, which is represented by the standard model, it is not considered that the knowledge network itself is intelligent, that is, there are two kinds of operations, one is to create a knowledge network, and the other is to use the knowledge network to obtain information. As the knowledge component can be calculated, the knowledge network obtained by the series and parallel connection can also be calculated, so it is convenient to carry out the knowledge computation.

In fact, the computation of knowledge network is widely used in reality. For example, all kinds of circuit boards are the series and parallel of electrical components, and the simulation system can be formed by the combination of basic components. We call this as professional application of knowledge. It is actually the application of the knowledge network in every professional field, which in turn shows that the standard model of knowledge representation to represent knowledge is more favorable.

Comparison Process. In addition to artificial agent, the internal structure of other agents is unknown, so we consider that the knowledge network of each agent is unknown. So in the knowledge communication, most of the situation is to exchange under the black box that need input and output to determine the content of knowledge.

In the process of knowledge communication, the comparison of knowledge network is very complex. In order to discuss conveniently, it is assumed that the comparison process is the process 
that one agent gives input, output and relationship and another agent constructs similar inner knowledge network from the information. For the knowledge network represented by the semantic network, the semantic network only represents the direct relationship between the two nodes, which is not easy to express multiple nodes and indirect relations. Another agent can directly establish the relationship between the nodes, or get the indirect relationship between them by reasoning. For example, agent A said "The ducks are oviparous", agent B could establish knowledge network directly: "The ducks are oviparous", or reasoning, if successful, establish the knowledge. If you do not succeed, then apply for additional input and output information.

For a knowledge network of standard model, if an agent gives the input, output and relationship, then another agent will try to use its own knowledge network to solve the problem firstly. If successful, accept it, else design a black box model as a knowledge component directly, namely receiving the input knowledge from the agent. You can also apply for additional input, output data, or make inference according to the inner knowledge network, accept or deny the input of knowledge finally.

Basic Running Process of Knowledge Communication. Knowledge communication process can be understood as the computation, comparison and construction of both agents through knowledge network. Suppose that the communication is in natural language, and the knowledge network is generated using standard model. In the following, a typical operation process of compared agent is given. The thought of basic operation flow is shown in Fig. 2.

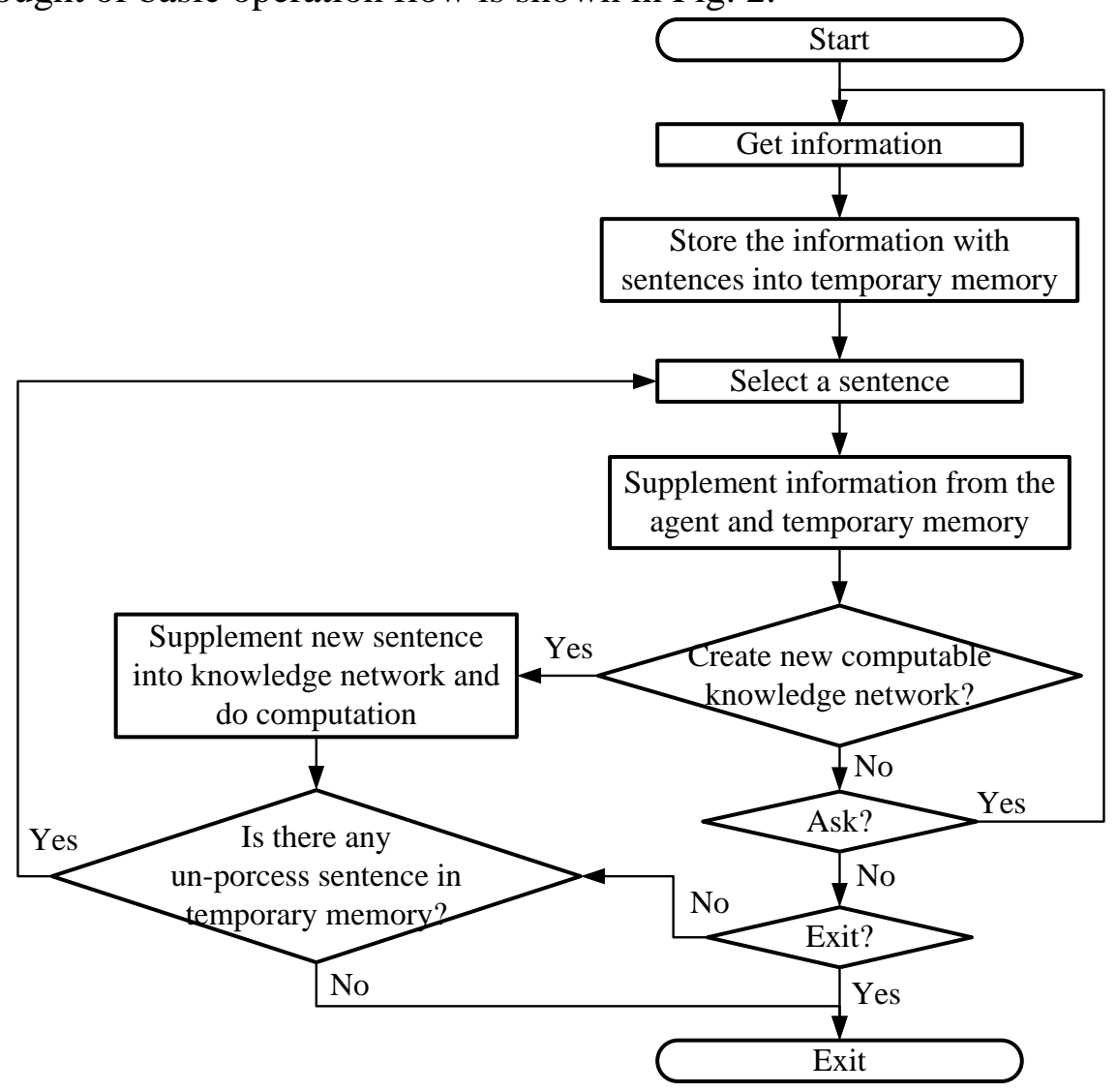

Fig. 2 basic running process

\section{Summary}

The power of knowledge is expressed by the computation of the knowledge network, and the effect of the application of knowledge will eventually come out as a result of the application of all kinds of artificial products created by human beings or all kinds of behaviors of man. But the computation of the knowledge network can not be separated from the construction of the knowledge network, that is, all kinds of artificial products or all kinds of behavior are made or formed under the guidance of the specific knowledge network. As human knowledge transmission can not be copied, 
so the role of knowledge communication is very important. The core of knowledge communication is the construction and computation of the knowledge network, and the construction of knowledge network needs higher intelligence. Therefore, it is of great significance to the representation and application of knowledge to study the construction and computation of knowledge system from the perspective of the creation and computation of knowledge network.

\section{References}

[1] Shi Zhongzhi. Advanced Artificial Intelligence [M]. Beijing : Science Press, 2011.7

[2] George F. Lunger. Artificial Intelligence : Structures and Strategies for Complex Problem Solving[M]. Pearson Education Limited 2002, 2005

[3] Joseph C. Giarratano, Gary D. Riley. Expert Systems : Principles and Programming[M]. Course Technology, a division of Thomson Learning, Inc., 2005

[4] Newell A, Simon H. A. A Program That Simulates Human Thought [M]. New York, USA: McCraw-Hill, 1963

[5] Feigenbaum E. A., Feldman J. Computers and Thought [M]. New York, USA : McGraw-Hill, 1963

[6] Simon H. A. The Sciences of Artificial [M]. Cambridge, USA: MIT Press,1969

[7] Newell A., Simon H. A. Human Problem Solving[M]. Englewood Cliffs, USA: Prentice-Hall, 1972

[8] Minsky M. L. The Society of Mind [M]. New York, USA: Simon and Schuster,1986

[9] Lin Chuang. Application of Petri nets to logical inference of horn clauses [J]. Journal of software, $1993,4(4): 32-37$

[10] Steven Walczak, Knowledge acquisition and Knowledge representation with class: the object-oriented paradigm [J]. Expert Systems with Applications, 1998,15 :235-244

[11]W. L. Xu, L. Kuhnert, K. Foster, et al, Object-oriented knowledge representation and discovery of human chewing behaviours $[\mathrm{J}]$. Engineering Applications of Artificial Intelligence, 2007,20:1000-1012

[12]Schank R. C. and Abelson R. Scripts, Plans, Goals and Understanding [M]. Hillsdale, NJ: Erlbaum, 1977

[13]Rukmi Sari Hartati, M. E.El-Hawary. A summary of application of Hopfield neural network to economic load dispatch [J]. 2000 Canadian Conference on Electrical and Computer Engineering, March 7-10, 2000

[14]Books R A. Intelligence without Representation [J]. Artificial Intelligence, 1991, 47(1/2/3): 139-160 